\title{
Effect of Strong Jammers on Passive Sonar Detection
}

\author{
Wang Yi-le ${ }^{1}$, Huangfu Li ${ }^{2, a}$, Zhuo Jie $^{1}$ \\ ${ }^{1}$ School of marine Science and Technology, Northwestern Polytechnical University, Xi'an 710072, \\ China \\ ${ }^{2}$ Science and Technology on Underwater Acoustic Antagonizing Laboratory, Beijing 100036, China \\ ahuangfl.seri@163.com
}

Keywords: broadband strong jammers; effective detection range; broadband array signal processing; direction of arrival

\begin{abstract}
The collection of target-radiated noise for passive sonar can be effected by broadband strong jammers, the detection range and accuracy will be reduced as a result of the appearance of jammer suppression area. In this paper, with the help of sonar equation and the theories of broadband array signal processing, the ship-radiated noise and ocean ambient noise are simulated to deduce the detection range of directional passive sonar under strong jammers, the direction of arrival and the output signal of passive sonar are also discussed.
\end{abstract}

\section{Introduction}

Generally, targets can be detected by passive sonar system through the detection of radiated noise. The receiver for the radiated noise of targets could be covered because of the existence of strong jammers with high power, this will lead to the appearance of jammers suppression area and targets couldn't be detected by passive sonar in it. The discussion about effect of strong jammers on passive sonar detection often guided by passive sonar equation, and it treats jammers as the background noise of the system, so the jammers suppression area can be deduced by pre-set detect threshold ${ }^{[1-5]}$. Since there is no combination of sonar signal processing theory in the discussion, the effect of strong jammers on system output signal waveform and the direction of arrival couldn't be given specifically. And therefore, a comprehensive and detailed analysis about the effect of strong jammers on passive sonar system couldn't be presented, which will not meet the needs of actual underwater acoustic antagonizing.

In this paper, effect of strong jammers on passive sonar detection is discussed based on the theories of broadband array signal processing and guided by passive sonar equation. The signal-level simulation and analysis is carried out by using simulated ship-radiated noise, ocean ambient noise and jammers signal. As a result, the detection range of directional passive sonar system under strong jammers is deduced, the effect on targets' signal waveform that system outputs and the estimation of direction of arrival are also discussed. So, a more comprehensive and detailed analysis about the effect of strong jammers on passive sonar system is given with the help of the whole discussion above.

\section{Concept of Operations}

The passive sonar equation that background noise is the major noise is:

$$
S L-T L-(N L-D I)=D T
$$

The normal detection range $R_{0}$ that sonar system can reach in different orientation under no jammers situation can be deduced from (1). Under the existence of jammers, the passive sonar equation will be adjusted to:

$$
S L-T L-\left[(N L-D I)+\left(S L i-T L i+D I\left(\theta_{i}\right)\right)\right]=D T
$$

$S L i$ is jammer source level, $T L i$ is the transmission loss of jammer, $\operatorname{DI}\left(\theta_{i}\right)$ is the space gain of sonar array for jammer, the ' + ' here is the combination of sound 
pressure. $S L i-T L i+D I\left(\theta_{i}\right)$ represent the energy leakage in the direction that strong jammers corresponding to the beam when the beam points to the target. This energy leakage makes the background noise of the working system increased, the SNR of input signal decreased immediately and the detect range of system suppressed. Detect range $R$ that sonar system in different orientation when jammers exist can be deduced from (2). The space width that $R<R_{0}$ represents is the effective suppression width of jammers.

While the energy of jammer is significantly higher than the background noise(up at least $6 \mathrm{~dB}$ ):

$$
S L i-T L i+D I\left(\theta_{i}\right)>(N L-D I)+6
$$

The sonar system is under the jammer dominant conditions, (2) can be simplified as

$$
S L-T L-\left(S L i-T L i+D I\left(\theta_{i}\right)\right)=D T
$$

While the strong jammer's nosie level at a certain distance is very low, or the strong jammer's nosie level is given, but jammers are too far from sonar result in a huge transmission loss, jammer noise is not huge than the background noise for sonar receiver in the conditions above:

$$
S L i-T L i+D I\left(\theta_{i}\right)<(N L-D I)
$$

Strong jammer no works or makes a little different, the maximum interference distance for a certain sonar which strong jammer works is presented as $R i_{\max }$.

$$
R i_{\max }=10^{\left(\frac{S L i+D I\left(\theta_{i}\right)-(N L-D I)}{20}\right)}
$$

From the point of view of the width of jammer suppress area, when the angle between strong jammer and submarine corresponding to the sonar is a bigger one than half of the sonar beam, the jammer is likely to fail. However, within a certain distance, jammers locate in the side lobe of passive sonar beam may also can cover up the ship-radiated noise from the targets locates in the main lobe in fact. As a result, there is still some suppress area outside the width of the sonar main lobe.

\section{Analysis of Jammer Works}

Using a 128 elements uniform linear array and spacing the array elements according to $500 \mathrm{~Hz}$ to set the property of sonar. The working bandwidth is from $10 \mathrm{~Hz}$ to $500 \mathrm{~Hz}$. The analysis of suppress property of strong jammer on passive sonar combines the sonar equation and broadband array signal processing theories.

\section{Ship-radiated Noise}

There are two line spectrums in the $20-200 \mathrm{~Hz}$ for ship-radiated noise. Ship-radiated noise decays by $-6 \mathrm{~dB} /$ oct after $200 \mathrm{~Hz}$ and the spectrum level at $1000 \mathrm{~Hz}$ is $110 \mathrm{~dB}$. The simulation of ship-radiated noise shows in Figure 1, the line spectrums locate in $50 \mathrm{~Hz}$ and $100 \mathrm{~Hz}$.
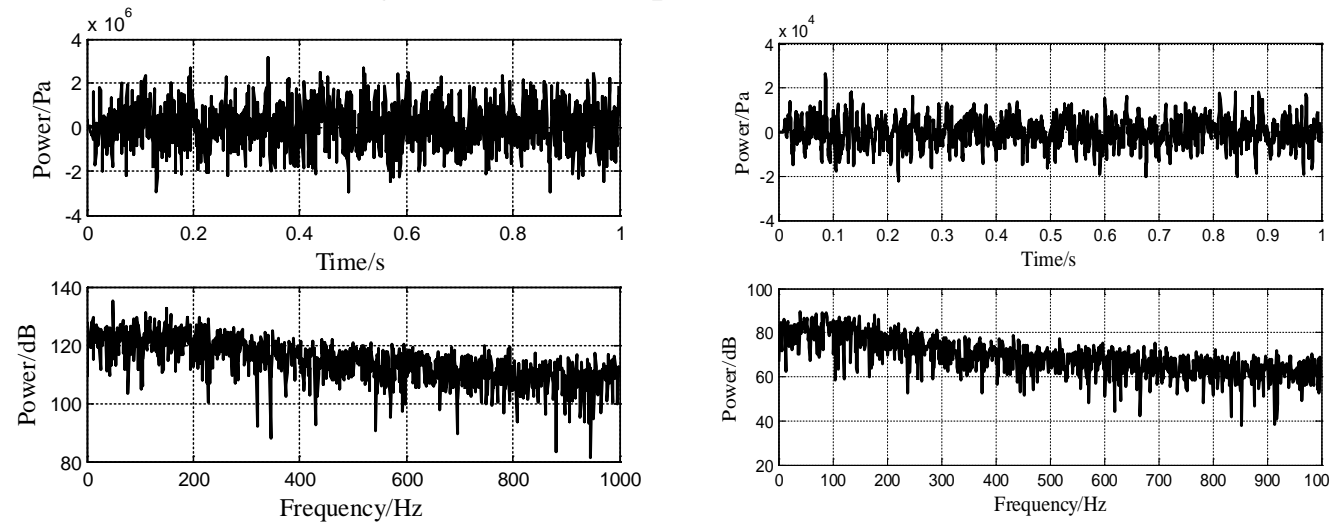

Fig.1 Ship-radiated noise

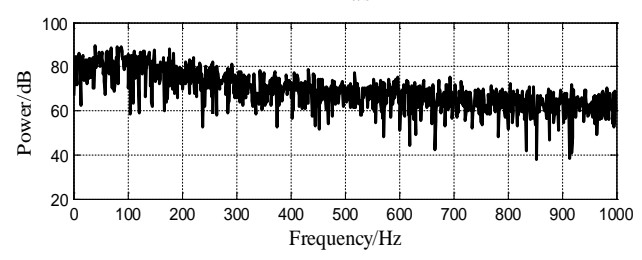

Fig.2 Ocean ambient noise

\section{Ocean Ambient Noise}

Noise spectrum decays by $-6 \mathrm{~dB} /$ oct after $100 \mathrm{~Hz}$ and the spectrum level at $1000 \mathrm{~Hz}$ is $64 \mathrm{~dB}$. The 
simulation of ocean ambient noise shows in Figure 2.

\section{Jammer Signal}

Jammer signal simulated using Gaussian white noise, and the sound source level in $10-500 \mathrm{~Hz}$ is 160dB.

\section{Principle of Jammer works}

The normal detection range is $R_{0}=11500 \mathrm{~m}$ for the given parameters of target and ambiance according to formula (1). The detection threshold of passive broadband sonar which uses non-correlation energy detector is set to $3 \mathrm{~dB}$. Jammer's parameters are set as: $S L i=160 \mathrm{~dB}$, $R i=5000 \mathrm{~m}, \theta_{i}=20^{\circ}$, sound source level of target and noise level is deduced by the simulated signal of ship-radiated noise and ambiance noise. The detection scope under a strong jammer is shown in Figure 3 (a) according to formula (2). Effective interference suppress area is changed with the parameter of jammer. The parameter of jammer is set as: $S L i=160 \mathrm{~dB}, R i=8000 \mathrm{~m}$, $\theta_{i}=-40^{\circ}$ in the Figure $3(\mathrm{~b})$.

The property of passive sonar detects target changes with the targets' location. Some cases are discussed as follow.

1)The spatial orientation of target is set as $10^{\circ}, R s=6000 \mathrm{~m}$, target locates in the detection scope of sonar according to Figure 3 (a). The power azimuth spectrum which multibeam sonar outputs is shown in Figure 4 (a). The time domain waveform and frequency spectrum of the output signal when sonar directs target is shown in Figure 4 (b).

Target locates in the detection scope according to Figure 4 (a), a $4.5 \mathrm{~dB}$ higher peak than the background noise appears in the target direction $\left(10^{\circ}\right)$ in power azimuth spectrum. Jammer makes no impact on system bearing estimation performance since $D T=3 \mathrm{~dB}$; As can be seen from Figure 4 (b), low frequency line spectrum at $50 \mathrm{~Hz}$ and $150 \mathrm{~Hz}$ that system outputs in the target's direction are obscured because of the existence of strong jammer.

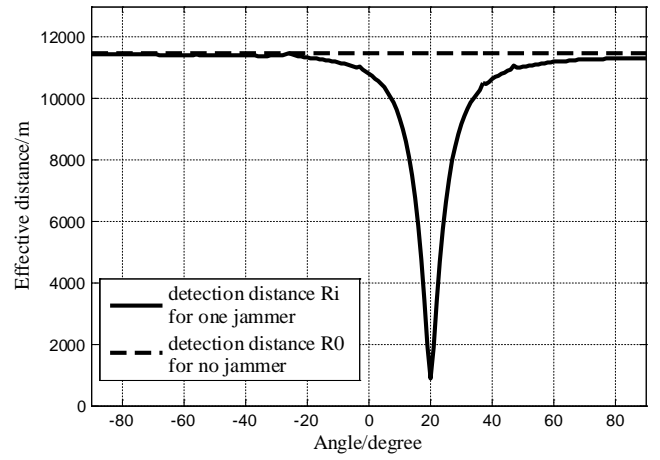

(a) Jammer No.1

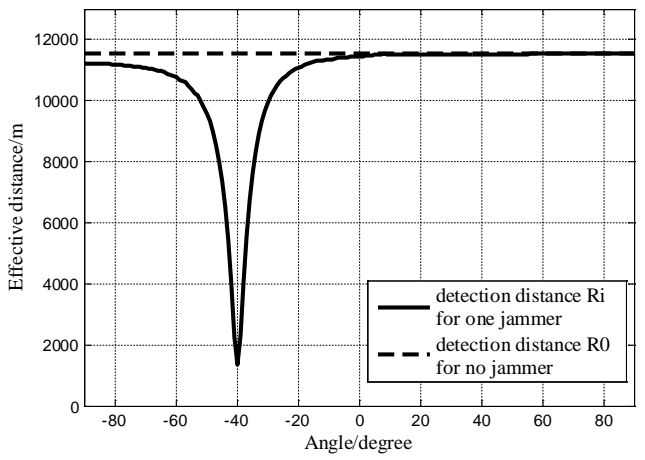

(b) Jammer No.2

Fig.3 Detection range under single jammer

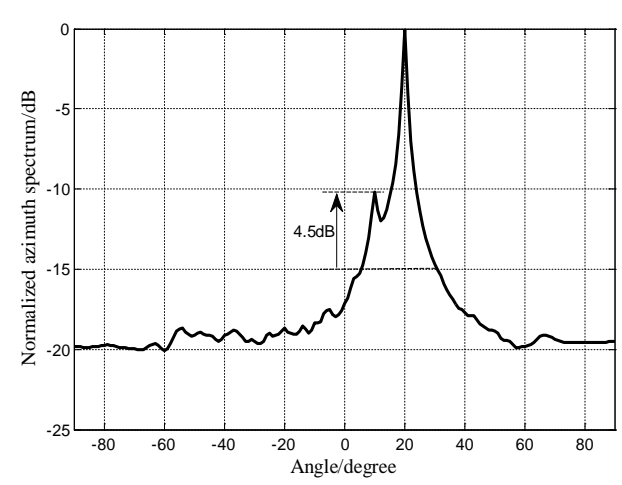

(a) Output signal in target direction
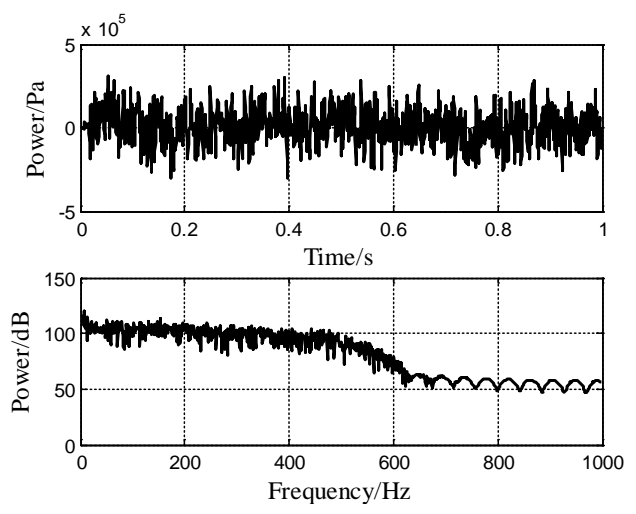

(b) Direction of arrival

Fig.4 Passive sonar system outputs ( $R s=6000 \mathrm{~m}$ ) 


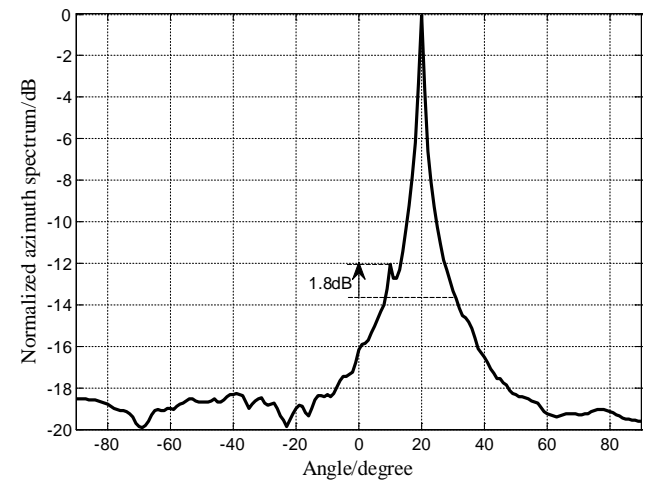

(a) Output signal in target direction
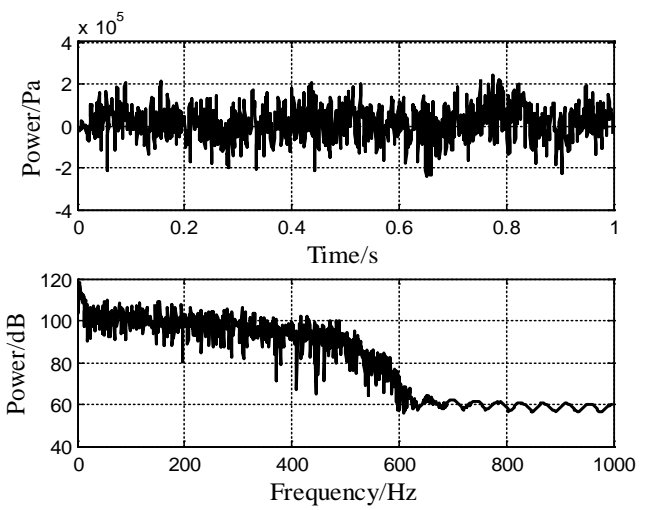

(b) Direction of arrival

Fig.5 Passive sonar system outputs ( $R s=10000 \mathrm{~m})$

2) The spatial orientation of target is set to $10^{\circ}, R s=10000 \mathrm{~m}$, target locates out of the detection scope of sonar according to Figure 3 (a). The power azimuth spectrum which multibeam sonar outputs is shown in Figure 5 (a). The time domain waveform and frequency spectrum of the output signal when sonar directs target is shown in Figure 5 (b).

As can be seen from Figure $5(\mathrm{a})$, a $1.8 \mathrm{~dB}$ higher peak appears in the target direction $\left(10^{\circ}\right)$ in the power azimuth spectrum since target locates out of the detection scope. Sonar does not detect the target due to the lack of $3 \mathrm{~dB}$, so jammer makes significant impact on system bearing estimation performance. As can be seen from Figure 5 (b), low frequency line spectrum at $50 \mathrm{~Hz}$ and $150 \mathrm{~Hz}$ that system outputs in the target's direction are obscured because of the existence of strong jammer.

\section{Conclusion}

In this paper, through combining sonar equation and broadband array signal processing theory, the principle of strong jammer impact passive sonar system is discussed. The signal-level simulation and analysis is carried out by using simulated ship-radiated noise, ocean ambient noise and jammers signal. As a result, the detection range of directional passive sonar system under strong jammers is deduced, the effect on targets' signal waveform that system outputs and the estimation of degree of arrival are also discussed. Summarizing the analysis of simulation, we can draw the following conclusions:

1) The effective suppression area that strong jammer makes to passive sonar is determined by the leakage energy that strong jammer in the sidelobe of sonar array, and the leakage energy is determined by the parameters of strong jammer which are sound source level, distance and orientation.

2) Low-frequency line spectral components are obscured because that the existence of strong jammer increase the background noise which has an impact on the frequency spectrum of target.

3) Strong jammer has a great impact on the performance of degree of arrival. The orientation of target can’t be accurately estimated when target locates in the jammer suppression area.

The analysis about how strong jammer affects passive sonar system has a certain reference value on how to reduce the impact that strong jammer makes to one's own detection equipment and how to use strong jammer to avoid the detection of enemy.

\section{References}

[1] CHEN chunyu. Anti-torpedo Technology[M]. National Defence Industry Press, 2006.

[2] ZHOU gang, CHEN qi. Warfare using efficiency evaluation of acoustics jamming equipment[J]. SHIP SCIENCE AND THECHNOLOGY, 2008,30(1):106-109.

[3] CHEN yichao. Effect of noise-jammer on suppressing sonar detection[J]. Technical Acoustics. 2011.30(1):74-77. 
[4] GAO xueqiang, YANG rijie. Modeling and Simulation of the nose-jammer combat effectiveness for anti-torpedo. Acoustics and Electronics Engineering[J]. 2009.3:11-13 93-100.

[5] WU peirong, GAO yuan. Research on evaluation method of countermeasure capability of low frequency noise jammer[J]. SHIP SCIENCE AND ECHNOLOGY. 2008.30(4):152-154. 